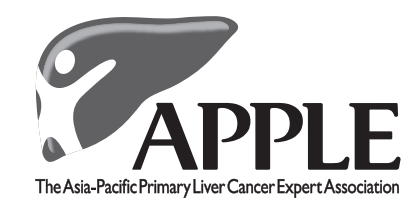

Steering Committee

Kwang-Hyub Han (Seoul)

Masatoshi Kudo (Osaka)

Sheng-Long Ye (Shanghai)

Ann-Li Cheng (Taipei)

President

Kwang-Hyub Han (Seoul)

President-Elect

Masatoshi Kudo (Osaka)

Secretary

Roonie T. Poon (Hong Kong)

Treasurer

Jong Young Choi (Seoul)

\section{Council Members}

Ann-Li Cheng (Taipei)

Pierce K.H. Chow (Singapore)

Namiki Izumi (Tokyo)

Jidong Jia (Beijing)

Norihiro Kokudo (Tokyo)

Kwan Sik Lee (Seoul)

Joong-Won Park (Goyang)

Michiie Sakamoto (Tokyo)

Jinsil Seong (Seoul)

\section{Honorary Chairpersons}

Ding-Shinn Chen (Taipei)

Miin-Fu Chen (Taipei)

Boo Sung Kim (Seoul)

Masamichi Kojiro (Kurume)

Hyuck Sang Lee (Seoul)

Masatoshi Makuuchi (Tokyo)

Young Myoung Moon (Seoul)

Kiwamu Okita (Yamaguchi)

Masao Omata (Yamanashi)

Jacqueline Whang-Peng (Taipei)

Yan Sun (Beijing)

Zhao-You Tang (Shanghai)

Meng-Chao Wu (Shanghai)

\section{Contact Information}

Department of Gastroenterology and Hepatology

Kinki University School of Medicine 377-2 Ohno-Higashi, Osaka Sayama Osaka, 589-8511 (Japan)

E-Mail: theliver@med.kindai.ac.jp

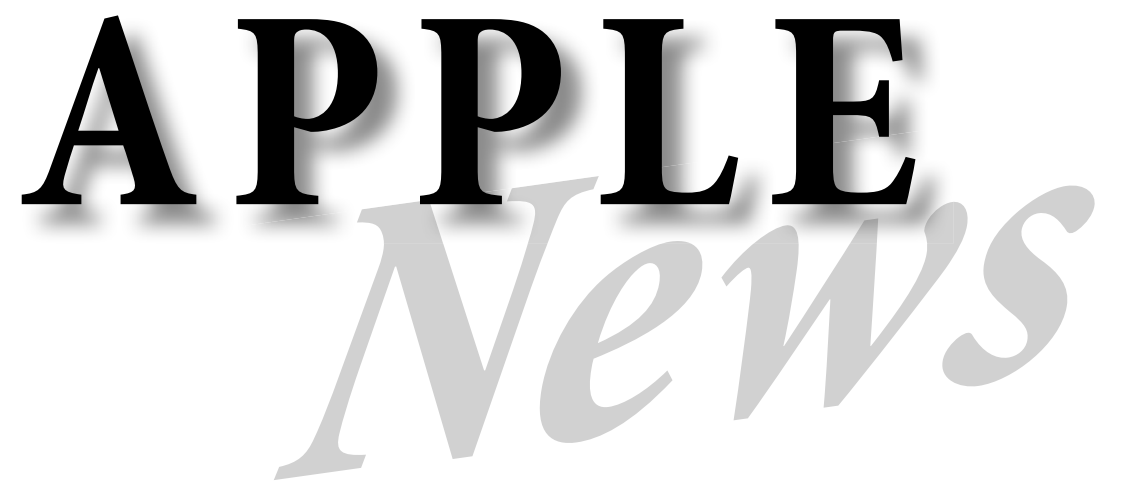

\section{Aims}

Primary liver cancer is an extremely malignant disease, and $80 \%$ of patients are found in Asian countries. The APPLE Meetings have been initiated to provide a platform for regional and international experts to exchange information on the latest advancements in primary liver cancer treatment. The meetings are aimed at bridging gaps in the basic science and clinical aspects of liver cancer among specialists, with the ultimate aim of disseminating the results of laboratory research and clinical trials in liver cancer among professionals.

\section{History}

The APPLE Meetings take place annually and were hosted in rotation by the Korean Liver Cancer Study Group, the Liver Cancer Study Group of Japan, and the Chinese Liver Cancer Study Group. The 1st meeting of the group took place in Incheon in 2010, the 2nd in Osaka in 2011, the 3rd in Shanghai in 2012, and the 4th in Busan in 2013. In 2014, APPLE changed its policy and decided to expand its congress venues from 3 countries to include all Asian countries. Therefore, the 5th APPLE Meeting was held in Taipei, Taiwan, in 2014.

\section{Next Meeting}

6th APPLE Meeting

Osaka, July 3-5, 2015

www.applecongress.org 